\title{
Exploring cosmic rays at the highest-energy frontier with the Pierre Auger Observatory
}

\author{
Carola Dobrigkeit ${ }^{1, a}$, on behalf of the Pierre Auger Collaboration ${ }^{2, b}$ \\ ${ }^{1}$ Instituto de Física Gleb Wataghin, Universidade Estadual de Campinas, 13083-859 Campinas, SP, Brazil \\ ${ }^{2}$ Observatorio Pierre Auger, Av. San Martín Norte, 304, 5613 Malargüe, Argentina
}

\begin{abstract}
The Pierre Auger Observatory studies the most energetic cosmic rays arriving at Earth, those with energies from $10^{17} \mathrm{eV}$ up to $10^{20} \mathrm{eV}$ and beyond. In continuous operation since 2004, the Observatory employs two complementary detection techniques for measuring air showers induced by those extremely energetic particles. For the past few years new detectors and techniques are being added in order to augment the sensitivity of the measurements. Data accumulated in ten years have led to major advances in our knowledge of the origin and nature of cosmic rays. We present a summary of the latest results for the spectrum of cosmic rays, their arrival directions and composition, as well as the challenges for the future operation of the Observatory.
\end{abstract}

\section{Introduction}

Since the discovery of cosmic rays a century ago, the study of these high-energetic particles represents a challenge. Arriving at Earth with energies from $10^{9} \mathrm{eV}$ up to $10^{20} \mathrm{eV}$ and above, their origin, propagation, and chemical composition have always piqued the interest of astrophysicists and particle physicists. A lot has been learned about these cosmic particles, mainly consisting of protons and atomic nuclei. The biggest question remains the elucidation of the characteristics of the particles at the upper end of spectrum. Understanding the acceleration processes up to macroscopic energies that they have undergone at their sources and the details of their cosmological propagation is already challenging. The flux of extremely energetic particles reaching Earth is very low, only about one such per square kilometer per year above $10^{19} \mathrm{eV}$, therefore requiring large detector arrays and long observation times to obtain any significant clues about these particles. Due to this low flux, it is not possible to measure their properties directly, and the study of ultrahigh-energy cosmic rays (UHECRs) is pursued through the measurement of the extensive air showers they induce when striking the atmosphere. For primary cosmic particles of $10^{18} \mathrm{eV}$, the first hadronic interactions with nuclei in the atmosphere occur at energies already beyond those attainable at terrestrial accelerators like the LHC, and their study allows one to establish a link between cosmic-ray physics and high-energy physics. Tracing back, from the detected secondary particles, the properties of the high-energy primary that initiated a shower, requires extrapolations of what we learned from accelerator experiments at energies up to two orders of magnitude less.

\footnotetext{
ae-mail: carola@ifi.unicamp.br

${ }^{b}$ Full author list:http://www.auger.org/archive/authors_2014_08.html
} 
The Pierre Auger Observatory faces the challenge of studying the origin and the nature of cosmic rays of ultrahigh energies. As the largest cosmic-ray observatory ever built, it covers an area of 3000 $\mathrm{km}^{2}$ in the Argentinian pampa, near the town of Malargüe, in the province of Mendoza. Situated at $32.5^{\circ} \mathrm{S}, 69.5^{\circ} \mathrm{W}$ and at $1400 \mathrm{~m}$ a.s.l., its location is privileged, allowing the observation of the region around the center of our Galaxy. The atmospheric conditions are favorable to the measurement of air showers through the fluorescence light emitted by nitrogen molecules after being excited by the billions of charged particles of an ultrahigh-energy air shower. Detecting UHECRs continuously since 2004, the Pierre Auger Observatory exploits two complementary detection techniques: a large surface array of 1600 water-Cherenkov detectors at the ground (SD) [1] and 24 fluorescence telescopes (FD) [2] disposed on the perimeter of the array and overlooking the huge atmospheric volume above it. The FD reaches a duty cycle of $13 \%$, operating only during clear moonless nights. The telescopes provide a means of obtaining an almost calorimetric measurement of the shower energy, and anchoring the energy calibration for the SD. With the FD the longitudinal development of extensive air showers in the atmosphere can be measured simultaneously (in "hybrid" mode), revealing clues about the mass composition of the primary particles. The surface array operates almost full time, registering air showers with a duty cycle of $98 \%$. From the lateral distribution of secondary particles at the ground, certain size parameters are measured for each air shower, and through the calibration of these parameters using data from both detectors, the energies of the corresponding primary particle can be inferred. The SD comprises two regular configurations, one covering the whole extension of the Observatory with water-Cherenkov detectors disposed on a triangular grid of $1500 \mathrm{~m}$ size, and a smaller infill area covering $30 \mathrm{~km}^{2}$, where 60 additional surface detectors are deployed to complete a subarray with half of that spacing. In the area of the infill, muon detectors (AMIGA) [3] and three additional telescopes with an elevated field of view (HEAT) [4] are installed, allowing an extension of the observed energy range down to $10^{17} \mathrm{eV}$. Over the last six years, some additional detectors have been added to the Observatory, exploring the detection of air showers through signals in the radio band (AERA) [5], and more recently, in the GHz band (AMBER, EASIER, MIDAS) [6]. Performing precision measurements of the fluorescence light in the atmosphere above the Observatory also requires a thorough monitoring of local atmospheric conditions [7]. The performance of all detectors is continuously monitored to assure high quality of the data [8].

\section{Energy spectrum}

The latest spectrum reported by the Pierre Auger Observatory includes four data sets collected until the end of 2012 [9]. These data sets are combined to compose the spectrum shown in figure 1: hybrid events measured with both the FD and the SD, events with the $750 \mathrm{~m}$ SD subarray and with the $1500 \mathrm{~m}$ full SD array, the latter two analyzed separately depending on their zenith angle being below or above $60^{\circ}$ (hereafter called "vertical" and "inclined", respectively). The flux $J(E)$ is multiplied by $E^{3}$ to favor an easy identification of features in the power-law spectrum $E^{-\gamma}$. A maximum-likelihood method is used to combine the four data sets, providing the relative normalization. Smearing corrections are applied to take into account possible bin-to-bin migrations. About 130,000 events compose this spectrum. The systematic uncertainty in the energy scale is $14 \%$ [10] and the energy resolution for events measured only with the SD is better than $12 \%$ above $10^{19} \mathrm{eV} \mathrm{[11].} \mathrm{Two} \mathrm{features} \mathrm{can} \mathrm{be} \mathrm{clearly}$ identified in the resulting spectrum: a sharp hardening of the spectrum at the so-called "ankle", and a suppression of the flux at the highest energies. At the energy of $5 \times 10^{18} \mathrm{eV}$ corresponding to the ankle, the power-law index changes from $3.23 \pm 0.07$ to $2.63 \pm 0.04$. Above $2 \times 10^{19} \mathrm{eV}$ the spectrum starts to deviate from this power law, being suppressed with respect to what would be expected by extrapolating the spectrum above the ankle. At $4 \times 10^{19} \mathrm{eV}$ the flux is half of that expected from that extrapolation. The significance of the suppression is $20 \sigma$. 

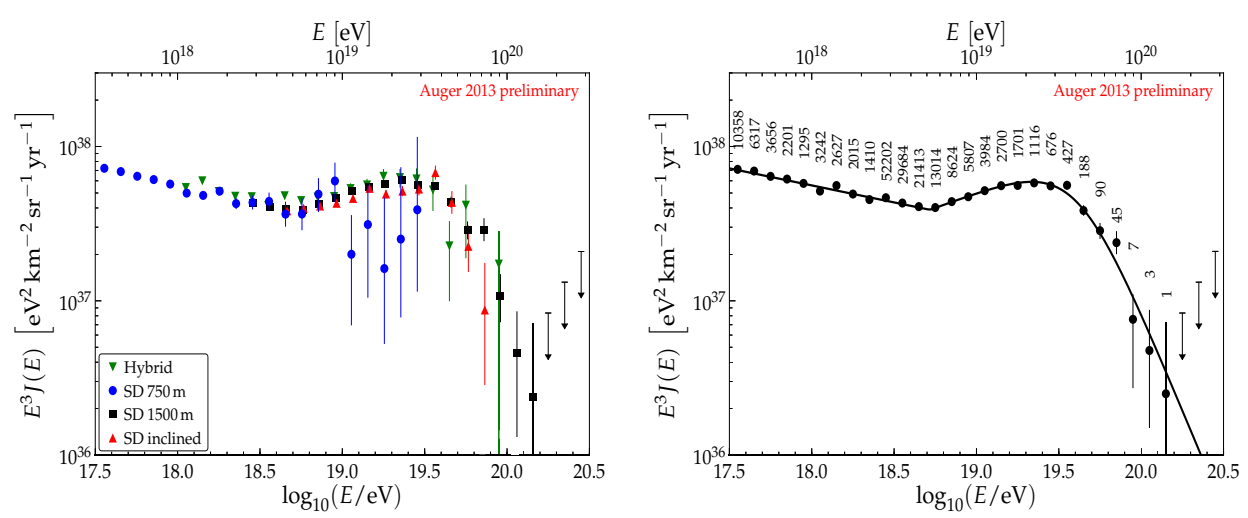

Figure 1. Left: The energy spectra obtained from hybrid events, and from data of the $750 \mathrm{~m}$ and $1500 \mathrm{~m} \mathrm{SD}$ arrays for vertical and inclined showers measured at the Auger Observatory. Right: The combined spectrum, with the number of events shown in each energy bin. The arrows represent upper limits at an $84 \%$ confidence level [9].

There are different scenarios to explain the causes of both the ankle and the suppression in the spectrum. Usually changes in the spectrum index are attributed to different origins, to changes in the cosmic-ray composition, to different processes occurring during the propagation of UHECRs from their sources to Earth, or else to a combination of these alternatives. The ankle can either indicate the transition from Galactic to extragalactic cosmic rays [12], or the distortion of an extragalactic proton-dominated spectrum as a consequence of $e^{ \pm}$pair production in interactions of these protons with photons from the cosmic microwave background (CMB). This latter scenario, known as the "dip model", requires a pure-proton composition, since heavier nuclei do not show this feature [13]. In this scenario the transition from Galactic to extragalactic cosmic rays would occur at lower energies, around $10^{17} \mathrm{eV}$. On the other hand, if the ankle were the indication of such a transition, cosmic rays of $10^{17} \mathrm{eV}$ would mostly originate from within our Galaxy, and their escape from it by diffusion would be expected to cause a dipole modulation in their angular distribution in this energy region.

The first prediction of a suppression of the flux at the highest energies dates from 1966, when soon after the discovery of the CMB radiation, Greisen [14] and, independently, Zatsepin and Kuz'min [15] foresaw this possibility as a consequence of energy losses caused by interactions of UHECRs with the $\mathrm{CMB}$ on their way to Earth. Protons of energies above $4 \times 10^{19} \mathrm{eV}$ can suffer pion photoproduction through the delta resonance, losing about $20 \%$ of their energy in each interaction. If UHECRs were heavy nuclei, they would also interact with $\mathrm{CMB}$ photons and lose part of their energy through photodisintegration. These processes limit the distances of possible astrophysical sources from us, constraining them to lie within a sphere of radius $\approx 100 \mathrm{Mpc}$. An alternative interpretation of the suppression is that the sources could be reaching the limit of their acceleration capability.

Details of the Auger spectrum were compared with theoretical predictions assuming different extragalactic astrophysical scenarios, pure-light or pure-heavy compositions, and two maximal acceleration energies at the sources, $10^{20}$ and $10^{20.5} \mathrm{eV}$. Auger flux measurements over the whole energy range from below the ankle up to the suppression region are in better agreement with predictions assuming a maximal acceleration energy of $10^{20} \mathrm{eV}$, and exclude a pure-iron composition [11].

The energy spectrum of UHECRs turns out to be most likely the result of multiple processes. In an intricate way, the spatial distribution of their sources, the acceleration process, the interactions suffered 
along their propagation, all affect the spectrum measured at Earth. Clearly, the measurement of the energy spectrum alone does not allow us to distinguish between the different scenarios mentioned above and complementary measurements of the mass composition are needed.

\section{Mass composition}

Identifying the mass of primary particles is one of the biggest challenges in the study of UHECRs. Most of the time, our knowledge relies on comparisons between measurements of certain shower observables and the predictions from shower simulations, which, in turn, use extrapolations of hadronic interaction models tuned to describe interactions at accelerator energies at least one order of magnitude less.

Due to the hybrid nature of the Pierre Auger Observatory, it is possible to measure air-shower observables that hold information about the mass of the primary particle that initiated the air shower. On the one hand, the fluorescence telescopes allow us to follow the longitudinal development of air showers in the atmosphere. The energy deposited in the atmosphere is predominantly due to the electromagnetic component of the shower cascade, depending on the air mass traversed, referred to as the slant depth $X$. Applying quality criteria in the selection of events, the mean atmospheric depth at which the showers in a certain energy range deposit the maximum energy in the atmosphere, $\left\langle X_{\max }\right\rangle$, and its fluctuations, $\sigma\left(X_{\max }\right)$, can be obtained. On the other hand, the time structure of particles reaching the surface detector array on the ground allows us to measure the distribution of muon production distances and from that distribution infer the slant depth at which the production of muons reaches its maximum, $X_{\max }^{\mu}$. The depths of both maxima are, of course, strongly correlated, mainly through the depth of the first interaction. At the same time, these measurements provide a means of testing and constraining models of hadronic interactions at the energies involved in the shower. Measuring the longitudinal profile of air showers, the FD is sensitive to the first interactions which occur near the top of the atmosphere. The SD, in measuring the particles reaching the ground, is sensitive to the whole hadronic cascade. The first two moments of the distributions of $X_{\max }$ and $X_{\max }^{\mu}$ are then compared to the results of Monte Carlo simulations of complete air showers, adopting for the generation of the hadronic interactions the models Sybill 2.1 [16], QGSJetII-04 [17] and EPOS-LHC [18]. The last two models already had their parameters tuned to recent data from LHC, and are extrapolated to the energies involved in air showers. Starting with the mean depth of shower maximum and its fluctuations, it is expected, from a simple shower model assuming that a nucleus can be treated as a superposition of its nucleons (and confirmed by results from simulations), that nucleus-induced showers develop higher in the atmosphere, resulting in lower $\left\langle X_{\max }\right\rangle$ than for proton-induced ones. The distribution of $\left\langle X_{\max }\right\rangle$ is also narrower for nucleus-induced showers than for proton-induced ones, resulting in smaller $\sigma\left(X_{\max }\right)$.

The measurement of the distributions of $X_{\max }$ of air showers with energy above $10^{17.8} \mathrm{eV}$ as measured with both the FD and the SD was recently published by the Auger Collaboration [19], presenting the distributions of $X_{\max }$ for eighteen energy bins. These distributions were obtained after applying rigorous selection criteria, yielding nearly bias-free measurements. The effects of acceptance and $X_{\max }$ resolution were carefully taken into account, both being parametrized as a function of energy and used in the deconvolution of the measured distributions to obtain the true distributions. The resolution in the measurement of $X_{\max }$ varies from $25 \mathrm{~g} \mathrm{~cm}^{-2}$ in the lower energy bin to $12 \mathrm{~g} \mathrm{~cm}^{-2}$ at the highest energy end. After correcting for acceptance and detection resolution, $\left\langle X_{\max }\right\rangle$ and $\sigma\left(X_{\max }\right)$ are compared to the predictions from simulations, assuming the three hadronic interaction models mentioned above, as shown in figure 2. Clearly, these observables do not follow a constant rate change with energy as predicted from simulations. Assuming that those models give a fair representation of 

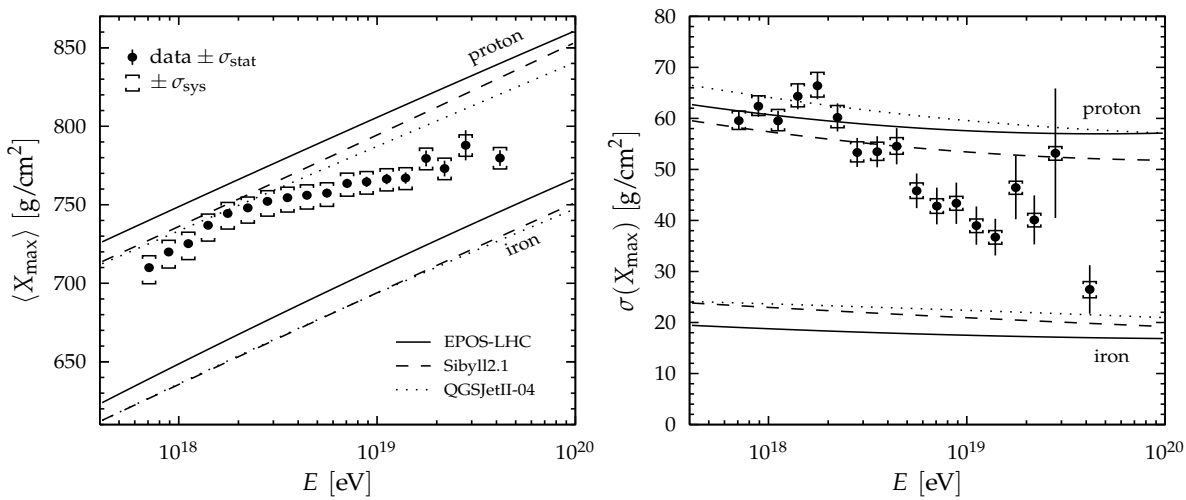

Figure 2. Evolution of $\left\langle X_{\max }\right\rangle$ (left) and $\sigma\left(X_{\max }\right)$ (right) as a function of energy. The error bars represent the statistical uncertainties. The systematic uncertainty in the energy scale is $14 \%$. The lines indicate the predictions from simulations using three different hadronic interaction models for proton- and iron-induced showers [19].

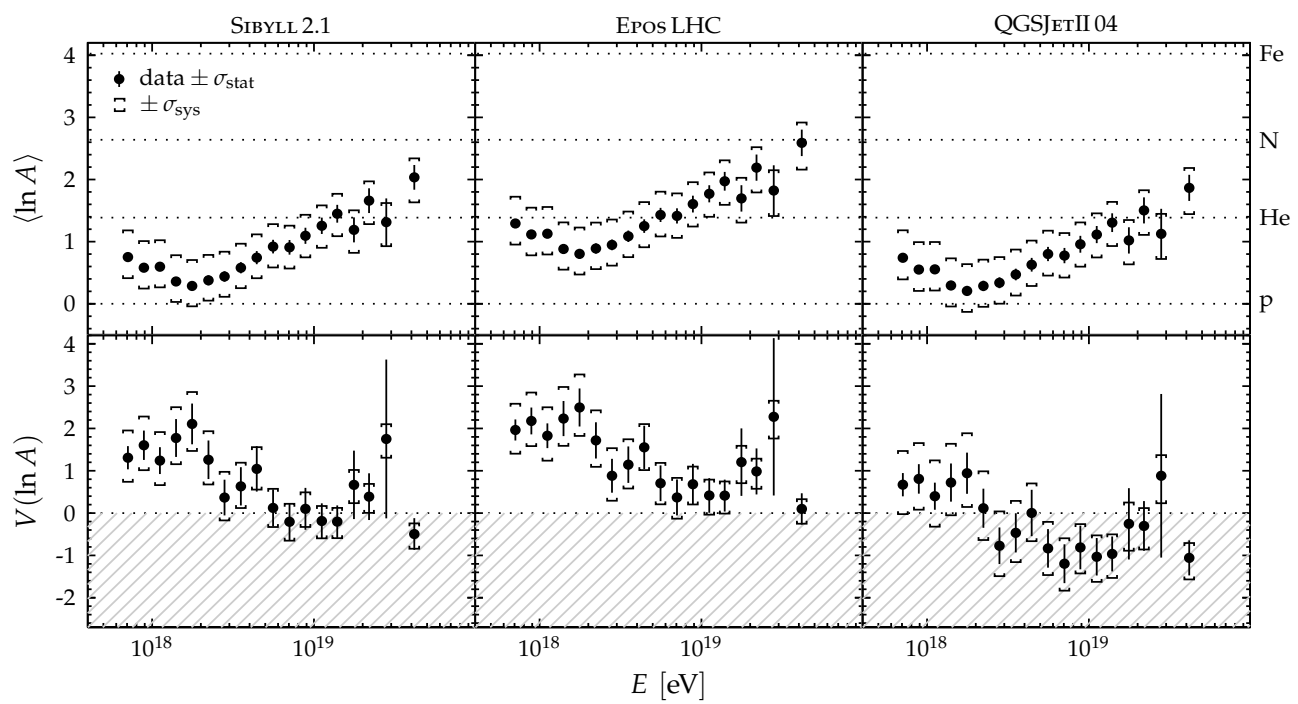

Figure 3. The mean logarithmic mass $\langle\ln A\rangle$ and its variance estimated from data using different hadronic interaction models [19].

the hadronic interactions in the shower, the measurements of both $\left\langle X_{\max }\right\rangle$ and $\sigma\left(X_{\max }\right)$ favor a change from a proton-like composition at around $10^{18.3} \mathrm{eV}$ towards a heavier one for energies above the ankle and an increase in the fraction of intermediate and heavier nuclei in the primary cosmic rays up to $10^{19.6} \mathrm{eV}$. From the measured values of both observables, and assuming the same interaction models, it is possible to determine the mean logarithmic mass $\langle\ln A\rangle$ of the primaries and its dispersion, where $A$ stands for the mass number of the primary particle [19]. In figure 3, the two first moments of the logarithmic mass distribution resulting from this procedure are shown and also indicate a change 
from a light to an intermediate-light composition, with $\langle\ln A\rangle$ increasing slowly above the energy of the ankle. Unphysical values are obtained in the case of QGSJetII-04 at higher energies.

The Pierre Auger Collaboration has also examined the implications of the measured $X_{\max }$ distributions for the composition of primary cosmic rays [20]. Rather than using only the first two moments of the distribution, its shape is used in this analysis, maximizing the information and reducing possible degeneracies in the interpretation. The whole hybrid $X_{\max }$ data set between $10^{17.8} \mathrm{eV}$ and $10^{20} \mathrm{eV}$ is used in the analysis, binning the showers in intervals of energy and $X_{\max }$. A template of $X_{\max }$ distributions for each primary species is created using the three hadronic interaction models EPOS-LHC, QGSJetII-04 and Sibyll. The simulated $X_{\max }$ for each species is determined by a quadratic interpolation around the peak as a function of slant depth. The template consists of the binned distribution after including effects of acceptance and measurement resolution. To carry out the comparison with the data, for a given energy bin the value in the template is weighted according to the fraction of each species. A binned maximum-likelihood method is then used to find the best fitting combination for these species. As a result of this analysis, it was shown that considering only a mixture of protons and iron nuclei, the fit qualities are poor throughout the whole energy range for all three interaction models. Although the peak data values lie between those for protons and iron nuclei, the resulting distributions are too narrow. When nitrogen nuclei are included in the analysis, the fit results acceptable only for EPOS-LHC. Further including helium nuclei results in all models being able to describe the data within systematic uncertainties over most of the energy range. Including the intermediate components brings the models into remarkable agreement in their predictions for the proton and iron nuclei contributions, despite large differences in the remaining composition. A substantial change in the proton fractions is observed over the whole energy range, dropping to near zero just above $10^{18} \mathrm{eV}$ with a possible resurgence at higher energies. In the region of the ankle, the fraction of protons in the primary flux is at the level of $\sim 90 \%$. Details of this analysis are reported in [20].

The analysis of $\left\langle X_{\max }\right\rangle$ and $\sigma\left(X_{\max }\right)$ relies on measurements of hybrid events, thus suffering from the lower number of events measured during the shorter duty cycle of the FD. The analysis of $\left\langle X_{\max }^{\mu}\right\rangle$ exploits the higher number of showers measured with the SD, and therefore also reaches higher energies. Results from simulations indicate that this quantity depends on the mass of the primary particle in a similar way as $X_{\max }$, its average and dispersion being smaller for heavier primaries compared to those obtained for lighter ones. An example of the $X_{\max }^{\mu}$ distribution for showers initiated by protons and iron nuclei of $3 \times 10^{19} \mathrm{eV}$ is shown in figure 4 (left), obtained with simulations adopting EPOSLHC as interaction model [21]. The analysis of muon production depths was performed on showers of energies above $2 \times 10^{19} \mathrm{eV}$ detected at the Auger Observatory from January 2004 up to December 2013. In the construction of the distributions of muon production depths, only muons from inclined showers with zenith angle between $55^{\circ}$ and $65^{\circ}$ and within a distance from the shower core between $1700 \mathrm{~m}$ and $4000 \mathrm{~m}$ were included, thus allowing a clear selection of the signal due to the muonic component, without any bias arising from the electromagnetic component. In figure 4 (right), the resulting $\left\langle X_{\max }^{\mu}\right\rangle$ are shown for five energy bins, and compared to predictions from simulations adopting QGSJetII-04 or EPOS-LHC. Both models predict a linear evolution of $\left\langle X_{\max }^{\mu}\right\rangle$ with the logarithm of the energy, although with a systematic difference of about $50 \mathrm{~g} \mathrm{~cm}^{-2}$. The data points are bracketed by the lines following predictions of the simulations for proton and iron primaries with the QGSJetII-04 model, and fall below those predicted from simulations with EPOS-LHC. Furthermore the data show a flatter trend than predicted for a pure-proton or a pure-iron composition. From these results, it is also possible to use the muon production depth as a tool to constrain hadronic interaction models.

Assuming that the shower simulations give a fair representation of the real processes occuring in air showers, it is possible to compare them to the data and derive clues about the primary mass composition. In a similar procedure as followed for $X_{\max }$, the measurements of $\left\langle X_{\max }^{\mu}\right\rangle$ can be converted 

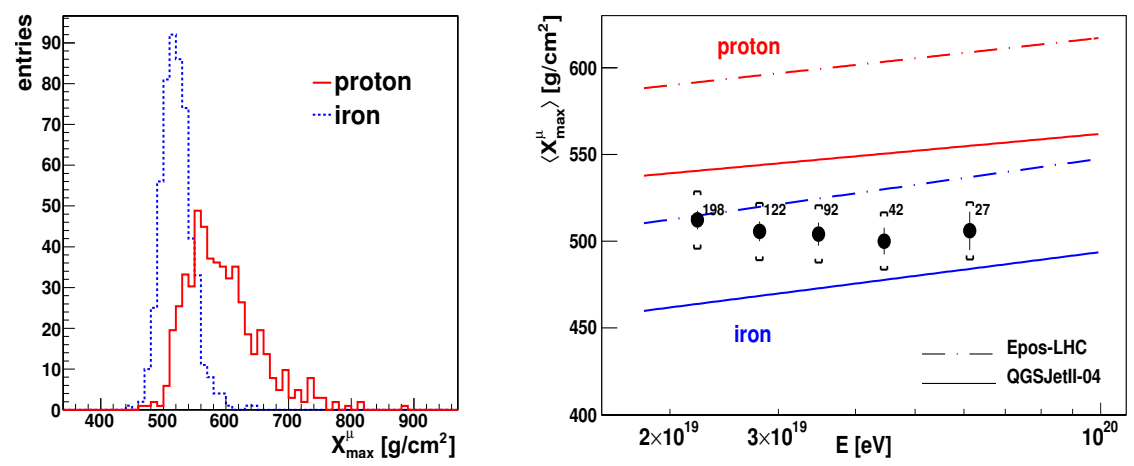

Figure 4. Left: $X_{\max }^{\mu}$ distributions in showers initiated by protons and iron nuclei of $3 \times 10^{19} \mathrm{eV}$ and angles between $55^{\circ}$ and $65^{\circ}$, simulated with EPOS-LHC. Right: Evolution of measured $\left\langle X_{\max }^{\mu}\right\rangle$ with energy, compared to the predictions of simulations using EPOS-LHC and QGSJetII-04 for proton and iron showers. The brackets represent the systematic uncertainty. The number of showers is shown for each energy bin [21].

to $\langle\ln A\rangle$ using the same interaction models. The outcome of the conversion is shown in figure 5 for the two models QGSJetII-04 and EPOS-LHC [21]. On the one hand, EPOS-LHC predicts primaries heavier than iron and the values of $\ln A$ obtained from the measurements of $X_{\max }$ and $X_{\max }^{\mu}$ are incompatible at a level of $2.5 \sigma$. On the other hand, although QGSJetII-04 yields compatible values for $\ln A$, it does not describe in a consistent way the first two moments of the $\ln A$ distribution obtained with the FD as already shown in figure 3. From these results one can thus conclude that none of the interaction models recently tuned to LHC data provides a consistent description of Auger data on the electromagnetic and muonic shower profiles.
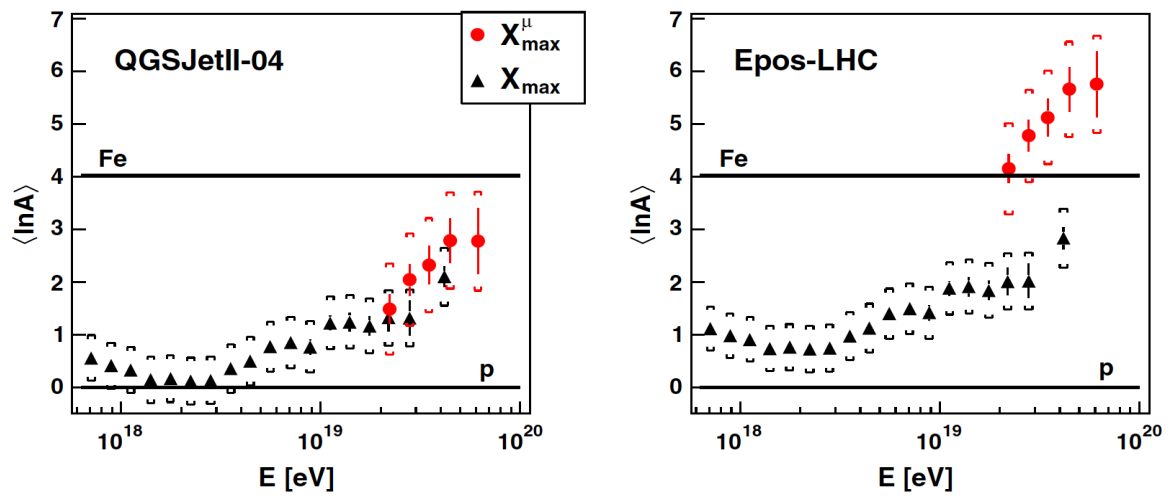

Figure 5. Conversion of $\left\langle X_{\max }\right\rangle$ (black triangles) and $\left\langle X_{\max }^{\mu}\right\rangle$ (red circles) to $\langle\ln A\rangle$, as a function of energy, for the hadronic interaction models QGSJetII-04 (left) and EPOS-LHC (right) [21].

\section{Arrival directions}

The arrival direction distribution is an important observable in the search for sources or source regions of UHECRs and for pointing out the transition from Galactic to extragalactic cosmic rays. A thorough 
analysis of the dipole anisotropy in the equatorial plane was performed in the energy region of the ankle with data from the $1500 \mathrm{~m} \mathrm{SD}$ array and from the $750 \mathrm{~m}$ infill array [22]. Different methods were applied in the analyses of data below and above $10^{18} \mathrm{eV}$, and the results are shown in figure 6 for the amplitude (left) and phase (right) of the first harmonic. For three energy bins above $10^{18} \mathrm{eV}$, the measured amplitudes have a probability of less than $1 \%$ of originating by chance from an isotropic distribution. Being more sensitive to a dipole anisotropy than the dipole amplitude, the phase angle of the first harmonic shows a smooth change in direction with increasing energy, changing from pointing to the Galactic center below $10^{18} \mathrm{eV}$ to the opposite direction at higher energy. A prescription is presently running to determine the statistical significance of this transition in the phase, and will end in 2015. The small dipole amplitude resulting from this analysis already challenges models in which cosmic rays are of Galactic origin up to the ankle.
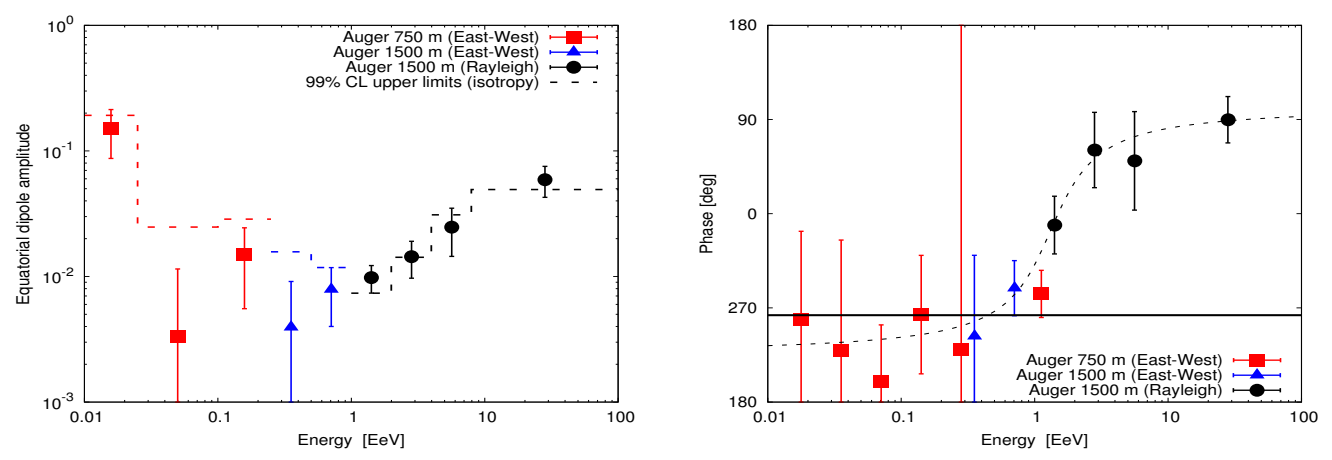

Figure 6. At left, the equatorial dipole amplitude of the reconstructed first harmonic in the arrival direction distribution is shown as a function of energy. The dashed line indicates the upper limit of the amplitude resulting from fluctuations of an isotropic distribution at a $99 \%$ confidence level. At right, the phase of the first harmonic as a function of energy is shown as reconstructed from data up to 2011. The solid line corresponds to the phase $\varphi=263^{\circ}$, and the dashed line is obtained from a fit assuming a logarithmic evolution of the tangent of the phase with energy [22].

An update of the correlation between arrival directions of the highest-energy cosmic rays and the directions of active galactic nuclei from the Véron-Cetty and Véron catalogue [23] was reported by the Pierre Auger Collaboration in 2010 [24]. In this update the fraction of correlation is estimated as $(33 \pm 5) \%$, compared to the prediction of $21 \%$ obtained assuming isotropy. The probability that this result would originate from a fluctuation from an isotropic distribution is $9 \times 10^{-3}$.

Recently, the Pierre Auger Collaboration and the Telescope Array Collaboration published the results for the dipole and quadrupole moments combining their data sets of arrival directions above $\approx 10^{19} \mathrm{eV}$ in a full-sky coverage [25]. The region of the sky for which the fields of view of both observatories overlap provides the cross-calibration factor for the different exposures and the compensation of systematic uncertainties due to different energy scales. No significant deviation from isotropy at any scale is found throughout the various analyses. Upper limits on the amplitude of the dipole and quadrupole moments are derived as a function of the direction in the sky, resulting between $7 \%$ and $13 \%$ for the dipole, and between $7 \%$ and $10 \%$ for a symmetric quadrupole. A sky map of the flux for the joint data set, for an expansion up to $\ell=4$, is displayed in equatorial coordinates in the left panel of figure 7, in units of $\mathrm{km}^{-2} \mathrm{yr}^{-1} \mathrm{sr}^{-1}$. For a better visualization of structures at intermediate angular scales, the sky map of the significance is presented in the right panel of figure 7 , smoothed out at a $15^{\circ}$ angular scale, showing regions of relative excesses and deficits. 

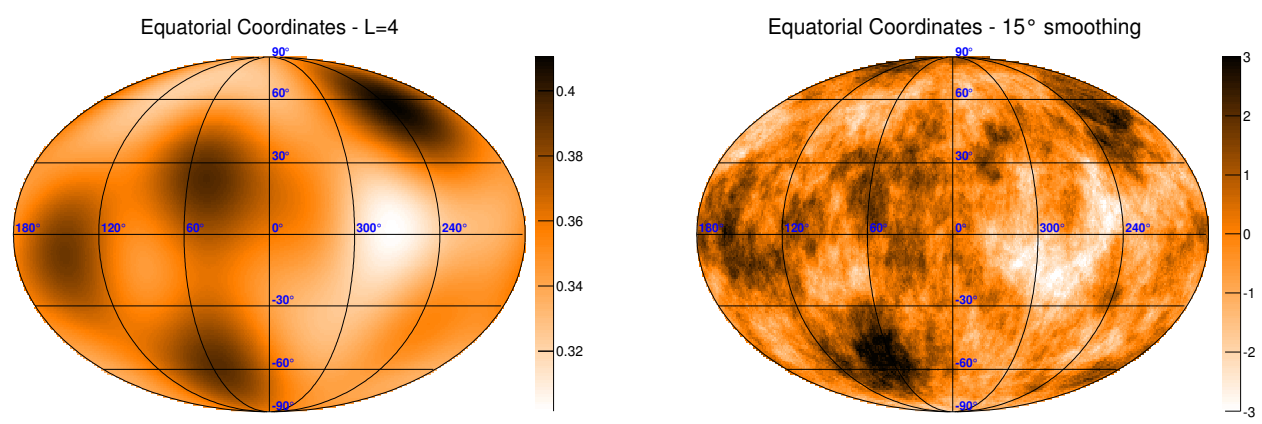

Figure 7. At left, the full-sky flux map is shown for a multipolar expansion up to $\ell=4$, in equatorial coordinates and units of $\mathrm{km}^{-2} \mathrm{yr}^{-1} \mathrm{sr}^{-1}$. At right, the sky map of the significance is shown, smoothed out at a $15^{\circ}$ angular scale [25].

Searches for primary photons [26], neutrons [27], and neutrinos [28] have also been performed by the Auger Collaboration. No significant evidence of detection has been found in any of these analyses, providing upper limits for photon fractions in the cosmic-ray flux, for the omnidirectional neutron flux, and for the neutrino flux in the field of view of the Observatory. These resulting limits also allow us to set constraints on some astrophysical scenarios of sources and propagation.

\section{Conclusion}

Results from the Pierre Auger Observatory clearly confirmed the sharp transition in the spectrum index at $4 \times 10^{18} \mathrm{eV}$ as well as the flux suppression above $5 \times 10^{19} \mathrm{eV}$. Based on these results alone, it is not possible to confirm whether the suppression is caused by the energy loss of cosmic rays during their propagation (the so-called GZK effect, after Greisen, Zatsepin, and Kuz'min), or is rather a signal of the maximum acceleration at their sources. To answer this question, the Auger Collaboration is currently proposing an upgrade of the Observatory, with additional detectors aiming at a better identification of the chemical composition of the primary particles, mostly increasing the mass sensitivity of the Observatory in the highest-energy region. To accomplish the increase in mass sensitivity, the detectors that will complement the surface detector array focus on improving the discrimination between the electromagnetic and muonic components of the showers. Measuring the identity of the primary cosmic ray on an event-by-event basis will help to address the issue of the maximum acceleration capability of the sources, if the suppression appears at energies proportional to the primary charge. It will also allow an improved study of anisotropies by selecting those primaries with a small charge and of hadronic interactions at center-of-mass energies an order of magnitude above those presently attainable at the LHC.

\section{Acknowledgement}

The successful installation, commissioning, and operation of the Pierre Auger Observatory would not have been possible without the strong commitment and effort of the technical and administrative staff in Malargüe. The author thanks the organizers of ICNFP 2014 and all colleagues in the Pierre Auger Collaboration. The author acknowledges the financial support from FAPESP (grant 2010/07359-6) and CNPq (Brazil). 


\section{References}

[1] J. Abraham et al. (The Pierre Auger Collaboration), Nucl. Instrum. Meth. A 523, 50 (2004)

[2] J. Abraham et al. (The Pierre Auger Collaboration), Nucl. Instrum. Meth. A 620, 227 (2010)

[3] F. Sánchez for the Pierre Auger Collaboration, Proc. 32nd ICRC 3,149 (2011)

[4] T. H. -J. Mathes for the Pierre Auger Collaboration, Proc. 32nd ICRC 3, 153 (2011)

[5] P. Abreu et al. (The Pierre Auger Collaboration), J. Instrum. 7, P10011 (2012)

[6] R. Gaïor for the Pierre Auger Collaboration, Proc. 33rd ICRC, arXiv:1307.5059 (2013)

[7] P. Abreu et al. (The Pierre Auger Collaboration), J. Instrum. 7, P09001 (2012)

[8] C. Bonifazi for the Pierre Auger Collaboration, Proc. 33rd ICRC, arXiv:1307.5059 (2013)

[9] A. Schulz for the Pierre Auger Collaboration, Proc. 33rd ICRC, arXiv:1307.5059 (2013)

[10] V. Verzi for the Pierre Auger Collaboration, Proc. 33rd ICRC, arXiv:1307.5059 (2013)

[11] A. Letessier-Selvon for the Pierre Auger Collaboration, Proc. 33rd ICRC, arXiv:1310.4620 (2013)

[12] A. M. Hillas, J. Phys. G Nucl. Part. 31, R95 (2005)

[13] V. Berezinsky, G. Gazizov, S. Grigorieva, Phys. Rev. D 74, 043005 (2006)

[14] K. Greisen, Phys. Rev. Let.16, 748 (1966)

[15] G. T. Zatsepin, V. A. Kuz'min, 1966, J. Exp. Theor. Phys. Let. 4, 78 (1966)

[16] E.-J. Ahn et al. Phys. Rev. D 8009400 (2009)

[17] S. Ostapchenko, Phys. Rev. D 83, 014018 (2011)

[18] T. Pierog et al., arXiv:1306.0121 [hep-ph] (2013)

[19] A. Aab et al. (The Pierre Auger Collaboration), accepted for publication in Phys. Rev. D (2014), arXiv: 1409.4809

[20] A. Aab et al. (The Pierre Auger Collaboration), accepted for publication in Phys. Rev. D (2014), arXiv: 1409.5083

[21] A. Aab et al. (The Pierre Auger Collaboration), Phys. Rev. D 90, 012012 (2014)

[22] I. Sidelnik, for the Pierre Auger Collaboration, Proc. 33rd ICRC, arXiv:1307.5059 (2013)

[23] M. -P. Véron-Cetty and P. Véron, Astron. Astrophys. 445, 773 (2006)

[24] P. Abreu et al. (The Pierre Auger Collaboration), Astropart. Phys. 34, 314 (2010)

[25] A. Aab et al. (The Pierre Auger and Telescope Array Collaborations), Astrophys. J. 794, 172 (2014)

[26] A. Aab et al. (The Pierre Auger Collaboration), Astrophys. J. 789, 160 (2014)

[27] A. Aab et al. (The Pierre Auger Collaboration), Astrophys. J. 760, 148 (2012)

[28] A. Aab et al. (The Pierre Auger Collaboration), Advances in High Energy Physics 2013, 708680 (2013) 